\title{
Novel Predictive Model for Adrenal Insufficiency in Dermatological Patients with Topical Corticosteroids Use: A Cross-Sectional Study
}

\author{
Suporntip Hintong ${ }^{1, *}$ \\ Phichayut Phinyo iD $2,3, *$ \\ Mati Chuamanochan ${ }^{4}$ \\ Mattabhorn Phimphilai' \\ Worapaka Manosroi iD' \\ 'Division of Endocrinology, Department \\ of Internal Medicine, Faculty of Medicine, \\ Chiang Mai University, Chiang Mai, \\ Thailand; ${ }^{2}$ Clinical Epidemiology and \\ Clinical Statistic Center, Faculty of \\ Medicine, Chiang Mai University, Chiang \\ Mai, Thailand; ${ }^{3}$ Department of Family \\ Medicine, Faculty of Medicine, Chiang Mai \\ University, Chiang Mai, Thailand; \\ ${ }^{4}$ Division of Dermatology, Department of \\ Internal Medicine, Faculty of Medicine, \\ Chiang Mai University, Chiang Mai, \\ Thailand
}

*These authors contributed equally to this work
Purpose: This study aimed to identify predictive factors and to develop a predictive model for adrenal insufficiency (AI) related to topical corticosteroids use.

Methods: The research was conducted using a cross-sectional design. Adult patients with dermatological conditions who had been prescribed topical steroids for at least 12 months by the dermatology outpatient departments of the Faculty of Medicine, Chiang Mai University from June through October 2020 were included. Data on potential predictors, including baseline characteristics and laboratory investigations, were collected. The diagnoses of AI were based on serum 8AM cortisol and low-dose ACTH stimulation tests. Multivariable logistic regression was used for the derivation of the diagnostic score.

Results: Of the 42 patients, $17(40.5 \%)$ had AI. The statistically significant predictive factors for AI were greater body surface area of corticosteroids use, age $<60$ years, and basal serum cortisol $<7 \mu \mathrm{g} / \mathrm{dL}$. In the final predictive model, duration of treatment was added as a factor based on its clinical significance for AI. The four predictive factors with their assigned scores were: body surface area involvement $10-30 \%(20),>30 \%(25)$; age $<60$ years old (15); basal serum cortisol of $<7 \mu \mathrm{g} / \mathrm{dL}$ (30); and duration of treatment in years. Risk of AI was categorized into three groups, low, intermediate and high risk, with total scores of $<25,25-49$ and $\geq 50$, respectively. The predictive performance for the model was 0.92 based on area under the curve.

Conclusion: The predictive model for AI in patients using topical corticosteroids provides guidance on the risk of AI to determine which patients should have dynamic ACTH stimulation tests (high risk) and which need only close follow-up (intermediate and low risk). Future validation of the model is warranted.

Keywords: adrenal insufficiency, topical corticosteroids, predictive model, skin diseases

\section{Introduction}

Topical corticosteroids are frequently used for inflammatory skin diseases owing to their anti-inflammatory and immunosuppressive effects. Common indications for use include diseases such as psoriasis, eczema, atopic dermatitis, and vitiligo. ${ }^{1}$ In clinical practice, a variety of delivery vehicles and potencies of topical corticosteroids are used. ${ }^{1}$ Prolonged and/or inappropriate use of topical corticosteroids can lead to adverse side effects. ${ }^{2}$ These adverse side effects can be categorized as cutaneous and systemic side effects. The most common cutaneous side effect is skin atrophy. Systemic side effects include hypothalamic-pituitary-adrenal (HPA) axis suppression, glaucoma, hyperglycemia and hypertension. ${ }^{3}$
Correspondence: Worapaka Manosroi Division of Endocrinology, Department of Internal Medicine, Faculty of Medicine, Chiang Mai University, Chiang Mai, Thailand

Tel +6653936453

Email worapaka.m@gmail.com 
One of the most worrisome adverse side effects from the use of topical corticosteroids is adrenal insufficiency (AI) resulting from HPA axis suppression. Topically applied corticosteroids can be absorbed systemically through the skin and can suppress the HPA axis. ${ }^{4-8}$ This adverse outcome, the inability to increase cortisol production after stress, can lead to adrenal crisis, which is potentially life-threatening. Tests that are normally used to diagnose or exclude AI include serum morning cortisol and the dynamic ACTH stimulation test. ${ }^{9}$

Secondary AI from percutaneous absorption of topical corticosteroids is less common than with parenteral or oral administration. The cumulative doses and the durations of oral corticosteroid therapy associated with HPA axis suppression have been well documented. ${ }^{10}$ Data regarding the dose and duration of oral corticosteroids and HPA axis suppression have similarly been well established. A study by Curtis et al reported that the use of oral prednisolone $>7.5 \mathrm{mg} /$ day for an extended period ( $>3$ weeks) was linked to this adverse event, and that the incidence increased with duration. ${ }^{10}$ However, corresponding data for topical corticosteroids has been limited. The degree of risk of HPA axis suppression from topical corticosteroids use is associated with the level of percutaneous absorption which, in turn, depends on numerous factors including the age of the patient (younger patients are more susceptible), body surface area treated, quantity of topical corticosteroids used, potency of the drug, duration of therapy, body region of application, the associated compounds used, eg, urea or salicylic acid, the characteristics of the diseased skin, the degree of impairment of skin integrity, and the coexistence of hepatic and/or renal disease. ${ }^{11-13}$ One study reported that HPA axis suppression occurs when high potency steroids are administered at a cumulative dose per week of $>50 \mathrm{~g}^{2}$

Presently, there is a lack of data on predictive factors for $\mathrm{AI}$ and no predicative model of the relationship between secondary AI resulting from HPA axis suppression and topical corticosteroids use. A simple predictive model which could help preclude and predict the risk of AI which incorporates both demographic and biochemical data could potentially reduce the number of dynamic ACTH stimulation tests performed. This study aimed to identify potential predictive factors and to design an easyto-use model for predicting the risk of AI following topical corticosteroids use in dermatological patients.

\section{Materials and Methods}

This cross-sectional study was conducted with 42 patients who were seen at the dermatology outpatient departments at the Faculty of Medicine, Chiang Mai University Hospital over a 5-month period (June - October 2020). The study protocol was approved by the Faculty of Medicine, Chiang Mai University, Ethical Committee (Ethical number: MED-2563-07037). Recruited participants were adult dermatological patients ( $\geq 18$ years) who had used topical corticosteroids for at least 12 months. Patients with pituitary or adrenal diseases, pregnant women and patients who had been treated with either systemic corticosteroids or other local corticosteroids were excluded. Those who meet all the inclusion criteria gave their informed consent prior to the study. This study was conducted in accordance with the Declaration of Helsinki.

\section{Adrenal Function Evaluation}

Adrenal function was evaluated by serum morning (8 AM) cortisol and the low-dose ACTH stimulation test. Patients were instructed to suspend use of topical corticosteroids for at least 24 hours before serum morning cortisol measurement and ACTH stimulation tests. In those with serum morning cortisol between 3 and $17.9 \mu \mathrm{g} / \mathrm{dL}$, ACTH stimulation tests were performed on the same day between 9-11AM to either exclude or diagnose AI. Serum cortisol concentrations were measured at 8 AM 0 (basal cortisol) as well as 20 and 40 minutes after $5 \mu \mathrm{g}$ ACTH was administered intravenously.

\section{Data Collection}

Epidemiological data collected included gender, age, blood pressure, underlying dermatologic diseases, other underlying diseases, body surface area involvement, sensitive area involvement, topical corticosteroid potency, amount and duration of topical corticosteroids use, symptoms of AI and the presence of Cushingoid features. Biochemical data included serum cortisol at $8 \mathrm{AM}, 0$ (basal cortisol) and at 20 and 40 minutes after ACTH intravenous injection, serum creatinine, electrolytes and albumin. Serum cortisol levels were measured by electrochemiluminescence assay (ECLIA) (Elecsys ${ }^{\circledR}$ Cortisol II assay, Roche Diagnostics GmbH, Mannheim, Germany).

\section{Definitions}

An 8 AM cortisol level of $<3 \mu \mathrm{g} / \mathrm{dL}$ or a peak serum cortisol level of $<18 \mu \mathrm{g} / \mathrm{dL}$ at 20 or 40 minutes after an 
ACTH stimulation test was defined as having AI. ${ }^{14}$ Sensitive area involvement included the axilla, groin, face and genitalia. Topical corticosteroids are classified by potency based on a skin vasoconstriction assay, and range from ultra-high potency (class I) to low potency (class VII). ${ }^{15}$ Since some patients had concurrently used more than one class of corticosteroids in one treatment period, the new variable potency dose time (summary of corticosteroids potency (I-VII) ${ }^{16}$ multiplied by total doses (mg) of corticosteroids use and multiplied by duration (months) of corticosteroids use) was created. Symptoms of AI included lethargy, nausea and vomiting, orthostatic hypotension and significant weight loss. Significant weight loss was defined as a loss of $5 \%$ of body weight in one month or a loss of $10 \%$ over a period of six months. ${ }^{17}$ Having Cushingoid features was defined as at least one of the excess glucocorticoid features, eg, easy bruising, facial plethora, proximal myopathy, striae, dorsocervical fat pad, facial fullness, obesity, supraclavicular fullness, hirsutism, decreased libido and menstrual abnormalities.

\section{Statistical Analysis}

All statistical analyses were performed using Stata 16 (StataCorp, College Station, Texas, USA). Categorical variables are reported as frequency and percentage, while continuous variables are reported as mean \pm standard deviation or median and interquartile range (IQR), according to their distribution. For univariable comparison, Fisher's exact probability test was used for categorical variables, and the independent $t$-test or the Mann-Whitney $U$-test was used for continuous variables. $p$-values less than 0.05 were considered statistically significant.

Multivariable logistic regression was used in the derivation of the prediction model for AI. Predictors with significant $\mathrm{p}$-values in the univariable analysis were included in the multivariable model. We also included age and treatment duration in the model due to the clinical significance of those factors. ${ }^{4,18}$ The clinical collinearity among the predictors was also evaluated before the selection of the predictors. We generated a weighted score for each predictor by dividing the logit coefficient of the predictor by the lowest coefficient in the model. The discriminative ability of the final multivariable model was assessed using the area under the receiver operating characteristics (ROC) curve. The calibration of the scores was evaluated using the Hosmer-Lemeshow goodness-of-fit test, where a p-value $>0.01$ was considered a good fit. For clinical applicability, the appropriate cut-off points for the scores were identified based on sensitivity and specificity. We identified one cut-off point with high sensitivity for ruling out AI and another cut-off point with high specificity for ruling in AI. The positive predictive value for each score category with its corresponding confidence interval were presented. A sample size of at least 25 patients with at least 5 patients with AI was estimated to give $80 \%$ power at the $5 \%$ significance level. ${ }^{4}$ There was no missing data in this study.

\section{Results}

Baseline characteristics and biochemical investigations are shown in Table 1. Forty-two patients with dermatological diseases were included in this study. Of these, 17 patients $(40.5 \%)$ had AI of whom $5(29.4 \%)$ were female. The mean age of the group was $56.5 \pm 15.4$ years, the mean duration of treatment was $10.1 \pm 6$ years, and the majority of patients had psoriasis $(n=14,82.4 \%)$. There was no significant difference in sex, age, duration of treatment, potency dose-time, comorbidities, or underlying skin disease between the AI and non-AI groups. The average body surface area of corticosteroids use was significantly higher in patients with AI than in the nonAI group $\left(27.5 \pm 18.7 \mathrm{~m}^{2}\right.$ and $10.7 \pm 11.7 \mathrm{~m}^{2}, \mathrm{p}<0.001$, respectively). Basal serum cortisol levels were significantly lower in the AI group $(6.52 \pm 4.04 \mu \mathrm{g} / \mathrm{dL})$ than in the non-AI group $(10.48 \pm 3.45 \mu \mathrm{g} / \mathrm{dL}, \mathrm{p} 0.003)$. Although lower serum morning cortisol levels were observed in the AI group, the difference was not statistically significant $(5.24 \pm 4.65 \mu \mathrm{g} / \mathrm{dL}$ vs $13.39 \pm 15.68 \mu \mathrm{g} /$ $\mathrm{dL}, \mathrm{p}=0.069)$. Three patients were identified as having Cushingoid features. All patients with Cushingoid features had AI.

Based on the multivariate logistic regression analysis (shown in Table 2), the significant predictive factors for AI in patients who used topical corticosteroids for more than 12 months were body surface area of corticosteroids use of $10-30 \%$ and $>30 \%$ (POR 18.9, $\mathrm{p}=0.042$, and POR $59.2, \mathrm{p}=0.035$, respectively), age less than 60 years (POR 13.8, $\mathrm{p}=0.04$ ), and basal serum cortisol of $<7$ $\mu \mathrm{g} / \mathrm{dL}$ (POR 131.5, $\mathrm{p}=0.003$ ). Only serum basal cortisol was included in the final multivariable model as there was clinical collinearity among serum morning cortisol and basal cortisol as well as 20- and 40-minute cortisol measurements.

Predictive risk score was created to determine the probability of patients having AI using the aforementioned three significant predictive factors from the multivariable analysis 
Table I Comparison of Clinical Characteristics Between Patients with a History of Topical Corticosteroids Use for at Least 12 Months Who Were Diagnosed with Adrenal Insufficiency and Those without Adrenal Insufficiency $(n=42)$

\begin{tabular}{|c|c|c|c|c|c|}
\hline \multirow[t]{2}{*}{ Clinical Characteristics } & \multicolumn{2}{|c|}{ Adrenal Insufficiency $(n=17)$} & \multicolumn{2}{|c|}{ Without Adrenal Insufficiency $(n=25)$} & \multirow[t]{2}{*}{ P-value } \\
\hline & $\mathbf{n}$ & (\%) & $\mathbf{n}$ & (\%) & \\
\hline Female & 5 & $(29.4)$ & 15 & $(60.0)$ & 0.066 \\
\hline Age $($ mean $\pm S D)$ & 56.5 & \pm 15.4 & 57.4 & \pm 14.7 & 0.838 \\
\hline Age $<60$ years & 12 & $(70.6)$ & 14 & $(56.0)$ & 0.518 \\
\hline Body mass index $\left(\mathrm{kg} / \mathrm{m}^{2}\right)($ mean $\pm \mathrm{SD})$ & 27.3 & \pm 5.4 & 24.8 & \pm 4.4 & 0.126 \\
\hline \multicolumn{6}{|l|}{ Skin diseases } \\
\hline Psoriasis & 14 & $(82.4)$ & 14 & $(56.0)$ & 0.375 \\
\hline Atopic dermatitis & 1 & $(5.9)$ & 2 & $(8.0)$ & \\
\hline Eczema & 1 & $(5.9)$ & 5 & $(20.0)$ & \\
\hline Others & 1 & $(5.9)$ & 4 & $(16.0)$ & \\
\hline \multicolumn{6}{|l|}{ Comorbidities } \\
\hline Diabetes mellitus & 5 & $(29.4)$ & 8 & $(32.0)$ & 1.000 \\
\hline Hypertension & 6 & $(35.3)$ & 11 & $(44.0)$ & 0.750 \\
\hline Cirrhosis & 1 & $(5.9)$ & 1 & $(4.0)$ & 1.000 \\
\hline Dyslipidemia & 7 & $(4 I .2)$ & 7 & $(28.0)$ & 0.508 \\
\hline Others & 6 & $(35.3)$ & 8 & $(32.0)$ & 1.000 \\
\hline \multicolumn{6}{|l|}{ Body surface area involvement } \\
\hline (\%) $($ mean $\pm S D)$ & 27.5 & \pm 18.7 & 10.7 & $\pm 1 \mathrm{I} .7$ & $<0.001$ \\
\hline$<10$ & 4 & $(25.5)$ & 14 & $(56.0)$ & 0.001 \\
\hline $10-30$ & 4 & $(23.5)$ & 10 & $(40.0)$ & \\
\hline$>30$ & 9 & $(52.9)$ & 1 & $(4.0)$ & \\
\hline Serum cortisol $0 \mathrm{~min}<7$ ( $\mu \mathrm{g} / \mathrm{dl})$ & 8 & $(61.5)$ & 2 & $(8.0)$ & 0.001 \\
\hline Treatment duration (year), (mean $\pm S D)$ & 10.1 & \pm 6.0 & 8.0 & \pm 5.4 & 0.244 \\
\hline Potency dose time, $($ mean $\pm S D)$ & 282.5 & \pm 338.6 & 221.0 & \pm 298.5 & 0.539 \\
\hline Median (IQR) & 147.4 & $66.2,303.5$ & 80.3 & $30.2,339.2$ & \\
\hline Cushingoid appearance & 3 & $(17.7)$ & 0 & (0) & 0.059 \\
\hline \multicolumn{6}{|l|}{ Symptoms of adrenal insufficiency } \\
\hline Lethargy & 0 & $(0)$ & I & $(4.0)$ & 1.000 \\
\hline Nausea and vomiting & 0 & $(0)$ & 0 & $(0)$ & NA \\
\hline Orthostatic hypotension & 0 & $(0)$ & 0 & $(0)$ & NA \\
\hline Weight loss & 1 & $(4.0)$ & 0 & (0) & 1.000 \\
\hline Serum morning cortisol $(\mu \mathrm{g} / \mathrm{dl}),($ mean $\pm \mathrm{SD})$ & 5.24 & 4.65 & 13.39 & 15.68 & 0.069 \\
\hline Serum basal cortisol ( $\mu \mathrm{g} / \mathrm{dl})$, (mean $\pm \mathrm{SD})$ & 6.52 & 4.04 & 10.48 & 3.45 & 0.003 \\
\hline Serum cortisol at $20 \min (\mu \mathrm{g} / \mathrm{dl}),($ mean $\pm \mathrm{SD})$ & 11.91 & 6.14 & 18.22 & 2.43 & 0.004 \\
\hline Serum cortisol at $40 \min (\mu \mathrm{g} / \mathrm{dl}),($ mean $\pm S D)$ & 13.94 & 6.71 & 20.76 & 2.33 & 0.002 \\
\hline Serum creatinine $(\mathrm{mg} / \mathrm{dl}),($ mean $\pm S D)$ & 1.04 & 0.30 & 1.80 & 4.20 & 0.477 \\
\hline Serum sodium $(\mathrm{mmol} / \mathrm{L}),($ mean $\pm \mathrm{SD})$ & 139.5 & 2.4 & $13 \mid .5$ & 35.2 & 0.422 \\
\hline Serum potassium $(\mathrm{mmol} / \mathrm{L}),($ mean $\pm \mathrm{SD})$ & 6.9 & 10.4 & 3.9 & 0.4 & 0.254 \\
\hline Serum bicarbonate $(\mathrm{mmol} / \mathrm{L}),($ mean $\pm \mathrm{SD})$ & 24.8 & 2.0 & 25.7 & 2.0 & 0.255 \\
\hline Serum albumin $(\mathrm{g} / \mathrm{dl})$ & 4.22 & 0.37 & 4.34 & 0.27 & 0.304 \\
\hline
\end{tabular}

Abbreviations: SD, standard deviation; IQR, interquartile range; NA, not applicable.

(Table 2). As previous studies have demonstrated that duration of treatment is a strong predictive factor for $\mathrm{AI}$ in corticosteroid users, ${ }^{4,18}$ this factor was also incorporated in the model. The transformed score for body surface area, age and basal serum cortisol had a range of 0 to 30 . For treatment duration, the transformed score was based on cumulative years of treatment. The total score was categorized into three groups: low, intermediate, and high risk (Table 3). 
Table 2 Multivariable Model for Prediction of Adrenal Insufficiency in Patients with a History of Topical Corticosteroids Use for at Least 12 Months $(n=38)$

\begin{tabular}{|c|c|c|c|c|c|}
\hline Predictors & POR & $95 \% \mathrm{Cl}$ & P-value & Coefficient & Weighted Score \\
\hline Age $<60$ years & 13.8 & $\mathrm{I} . \mathrm{I}, \mathrm{I} 68.4$ & 0.040 & 2.62 & 15 \\
\hline Serum cortisol at $0 \mathrm{~min}<7 \mu \mathrm{g} / \mathrm{dL}$ & 131.5 & $5.3,3242.6$ & 0.003 & 4.88 & 30 \\
\hline \multicolumn{6}{|l|}{ Body surface area involvement } \\
\hline$<10 \%$ & Ref & Ref & & Ref & 0 \\
\hline $10-30 \%$ & 18.9 & I.I, 320.9 & 0.042 & 2.94 & 20 \\
\hline$>30 \%$ & 59.2 & $1.3,2611.6$ & 0.035 & 4.08 & 25 \\
\hline \multirow[t]{2}{*}{ Treatment duration (years) } & 1.17 & $0.9,1.5$ & 0.164 & 0.16 & I $x$ year \\
\hline & & & & Total & $90+$ year \\
\hline
\end{tabular}

Abbreviations: $\mathrm{Cl}$, confidence interval; POR, prevalence odds ratio; Ref, reference.

Table 3 Accuracy of the Score to Rule in and Rule Out Adrenal Insufficiency in Patients with a History of Topical Corticosteroids Use for at Least 12 Months $(n=38)$

\begin{tabular}{|l|c|c|c|c|}
\hline Risk Level of Adrenal Insufficiency & Cut-Off Point & Al (n = I3) & Non-AI (n = 25) & Positive Predictive Value (95\% CI) \\
\hline Low & $<25$ & 13 & 0 & $0(0,24.7)$ \\
Intermediate & $25-49$ & 7 & 12 & $36.8(16.3,61.6)$ \\
High & $\geq 50$ & 6 & 0 & $100(54.1,100)$ \\
\hline
\end{tabular}

The cut-off point of $\geq 50$ suggests high risk for developing AI with a sensitivity of $46.2 \%$ and a specificity of $100 \%$, a score of $<25$ suggests a low risk with a sensitivity of $100 \%$ and a specificity of $52 \%$, and a score between 25 and 49 indicates an intermediate risk of having AI. The ROC curve for the model assessing predictive performance which included all significant factors had an AuROC of 0.92 (Figure 1). The Hosmer-Lemeshow goodness-of-fit test revealed non-statistically significant results $(\mathrm{p}=0.599)$, indicating that our newly derived scoring system fits the data well.

\section{Discussion}

The present study proposes an easy-to-use predictive model for AI following topical corticosteroids use in dermatological patients based on demographic and biochemical factors. The accuracy of the model shows an excellent diagnostic accuracy of $92 \%$ based on AuROC. Currently, the diagnosis of $\mathrm{AI}$ in dermatological patients with topical corticosteroids use involves multiple steps including screening for serum morning cortisol followed by dynamic ACTH stimulation testing. The proposed simple predictive model, which requires only three demographic data items (age, body surface area of corticosteroids use, duration of use) and one biochemical test (serum basal cortisol), could potentially reduce the number of dynamic ACTH stimulation tests performed, resulting in cost- and time-saving for both patients and health-care facilities.

Based on the proposed cut-off points, we suggest screening of individuals at high risk for having AI, including serum morning cortisol and the ACTH stimulation tests to confirm a diagnosis of AI. If there is evidence of AI, the patient should

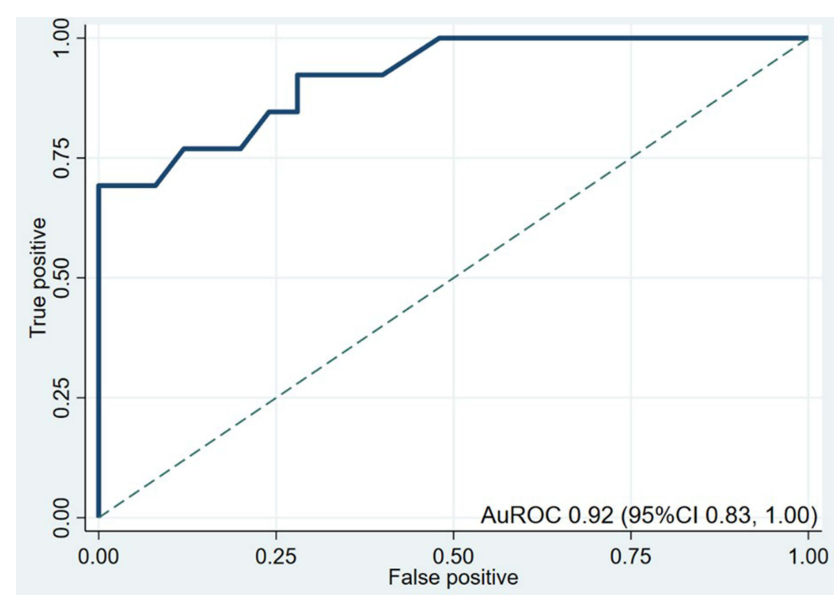

Figure I Model discrimination via receiver operating characteristic curve in patients with a history of topical corticosteroids use for at least 12 months $(n=42)$. 
begin to receive treatment for AI to reduce future complications. For those in the low-risk group, only clinical follow-up should be carried out. In the intermediate-risk group, we recommend regular and close biochemical follow-up including serum morning cortisol and clinical follow-up for signs and symptoms of AI. Signs and symptoms that should raise a high index of suspicion for AI include significant weight loss, nausea and/or vomiting, orthostatic hypotension and lethargy. However, this proposed predictive model was studied in adults and cannot simply be generalized and extrapolated to children or infants.

In our study, $40.5 \%$ of the patients were determined to have AI. A previous meta-analysis by Broersen et al reported the percentage of patients with AI secondary to all potencies of topical corticosteroids based on a review of 15 studies was $4.7 \%, 95 \% \mathrm{CI}(1.1-18.5 \%){ }^{19}$ The higher prevalence of $\mathrm{AI}$ in our study could be a result of differences in patients' baseline characteristics, eg, duration of treatment, corticosteroids potency and body surface area involvement.

In the predictive model, we incorporated both clinical and biochemical factors which are easy to obtain in actual clinical practice. Some of those predictive factors have been previously reported to be linked to AI. Body surface area of corticosteroids use larger than $10 \%$ found to be significantly related to AI, especially in patients with a lesion area of over $30 \%$. This finding is consistent with a study by Kerner et al which suggests the extent of surface area to which the corticosteroids are applied may influence absorption of the drug. ${ }^{20}$ Regarding the age of the patients, our study found that individuals over 60 years old tended to be at high risk of AI following topical corticosteroids therapy. The underlying explanation is that the stratum corneum acts as a rate-limiting barrier to percutaneous absorption as the stratum corneum in younger individuals is thinner than in older people. Diminished effectiveness of topical corticosteroid treatment in older people was demonstrated in a study by Malzfeldt et al. ${ }^{21}$ Even though serum basal cortisol is not recommended as a standard test to diagnose AI, a prior study reported that it can be considered as an alternative choice to diagnose AI when serum morning cortisol results are not available. In fact, it has been reported that there is no difference in diagnostic accuracy between serum morning cortisol and basal cortisol ${ }^{22}$ which supports our finding that serum basal cortisol $<7 \mu \mathrm{g} / \mathrm{dL}$ is one of the significant factors related to AI.

The final model found no statistically significant relationship between the incidence of $\mathrm{AI}$ and the duration of corticosteroids treatment. However, we decided to include this factor in the final model since previous publications have reported that the duration of treatment is a relevant risk factor for developing AI following continuous topical corticosteroids use. The duration of AI events has been reported to vary between 2 weeks to 18 months., 48 Additionally, a case report of AI demonstrated that 5 years of topical corticosteroids use can cause AI. ${ }^{6}$ Together, this suggests that patients with a longer duration of topical corticosteroids use are at increased risk of AI, especially those who also have other risk factors. Although both potency and dosage of topical corticosteroids have been reported to be significantly linked to HPA axis suppression, the present study found only a non-significance link. This could be the result of the small sample size as well as of other factors, eg, body surface area involvement and serum cortisol levels, which could have masked the association between potency and dosage of topical corticosteroids with HPA suppression.

To the best of our knowledge, this study is the first to use these novel predictive factors to develop a predictive model for AI in patients using topical corticosteroids. This model has multiple potential implications. First, the model uses clinical and biochemical factors which are obtainable in many institutes. Second, the model's risk score provides good diagnostic accuracy in terms of both sensitivity and specificity. Finally, each of the predictive factors in the model has an underlying pathophysiological explanation and is not due simply to chance.

There are some limitations in this study. First, the sample size is relatively small, although it does offer sufficient statistical power for each of the predictive factors. Second, further external validation is needed to validate the predictive performance of the model. Third, the cut-off level of serum cortisol after ACTH stimulation test was based on the older generation of ECLIA assay. There was a study proposed that the cut-off for serum cortisol in the newer generation of cortisol assay should be lower $(\sim 14-15 \mu \mathrm{g} / \mathrm{dL})$ than the previous one $(18 \mu \mathrm{g} / \mathrm{dL}) .{ }^{23}$ However, this proposed cut-off has not yet been established in the current guideline for AI. In the future, if the newer cut-off for serum cortisol will have been employed in the standard guideline, our predictive model may lead to overdiagnosis of AI.

\section{Conclusions}

The proposed predictive model uses both demographic and biochemical factors to determine the risk of AI in dermatological patients following topical corticosteroids use with a high level of diagnostic accuracy. This model has 
advantages in terms of a reduction in the number of dynamic ACTH stimulation tests needed, thus saving time and resources. Additionally, it can provide guidance to clinical practitioners regarding which patients should be closely followed up for development of AI. Future external validation of this predictive model is warranted.

\section{Acknowledgments}

The authors are grateful to Lamar G. Robert, PhD and Chongchit S. Robert, PhD for editing the manuscript.

\section{Disclosure}

The authors report no conflict of interest in this work.

\section{References}

1. Ference JD, Last AR. Choosing topical corticosteroids. Am Fam Physician. 2009;79(2):135-140.

2. Hengge UR, Ruzicka T, Schwartz RA, Cork MJ. Adverse effects of topical glucocorticosteroids. J Am Acad Dermatol. 2006;54(1):1-15; quiz 16-8. doi:10.1016/j.jaad.2005.01.010

3. Rathi SK, D'Souza P. Rational and ethical use of topical corticosteroids based on safety and efficacy. Indian J Dermatol. 2012;57 (4):251-259. doi:10.4103/0019-5154.97655

4. Carruthers JA, August PJ, Staughton RC. Observations on the systemic effect of topical clobetasol propionate (Dermovate). $\mathrm{Br}$ Med J. 1975;4(5990):203-204. doi:10.1136/bmj.4.5990.203

5. Staughton RC, August PJ. Cushing's syndrome and pituitary-adrenal suppression due to clobetasol propionate. $\mathrm{Br}$ Med J. 1975;2 (5968):419-421. doi:10.1136/bmj.2.5968.419

6. Young CA, Williams IR, MacFarlane IA. Unrecognised Cushing's syndrome and adrenal suppression due to topical clobetasol propionate. Br J Clin Pract. 1991;45(1):61-62.

7. Abma EM, Blanken R, De Heide LJ. Cushing's syndrome caused by topical steroid therapy for psoriasis. Neth J Med. 2002;60(3):148-150.

8. Böckle BC, Jara D, Nindl W, Aberer W, Sepp NT. Adrenal insufficiency as a result of long-term misuse of topical corticosteroids. Dermatology. 2014;228(4):289-293. doi:10.1159/000358427

9. Ospina NS, Al Nofal A, Bancos I, et al. ACTH stimulation tests for the diagnosis of adrenal insufficiency: systematic review and meta-analysis. J Clin Endocrinol Metab. 2016;101(2):427-434. doi:10.1210/jc.2015-1700
10. Curtis JR, Westfall AO, Allison J, et al. Population-based assessment of adverse events associated with long-term glucocorticoid use. Arthritis Rheum. 2006;55(3):420-426. doi:10.1002/art.21984

11. Brazzini B, Pimpinelli N. New and established topical corticosteroids in dermatology: clinical pharmacology and therapeutic use. Am J Clin Dermatol. 2002;3(1):47-58. doi:10.2165/00128071-20020301000005

12. Dhar S, Seth J, Parikh D. Systemic side-effects of topical corticosteroids. Indian $J$ Dermatol. 2014;59(5):460-464. doi:10.4103/0019-5154.139874

13. Levin C, Maibach HI. Topical corticosteroid-induced adrenocortical insufficiency: clinical implications. Am J Clin Dermatol. 2002;3 (3):141-147. doi:10.2165/00128071-200203030-00001

14. Bornstein SR, Allolio B, Arlt W, et al. Diagnosis and treatment of primary adrenal insufficiency: an endocrine society clinical practice guideline. $J$ Clin Endocrinol Metab. 2016;101(2):364-389. doi: $10.1210 /$ jc. $2015-1710$

15. Goa KL. Clinical pharmacology and pharmacokinetic properties of topically applied corticosteroids. A review. Drugs. 1988;36(Suppl 5):51-61. doi:10.2165/00003495-198800365-00011

16. Davallow Ghajar L, Wood Heickman LK, Conaway M, Rogol AD. Low risk of adrenal insufficiency after use of low- to moderate-potency topical corticosteroids for children with atopic dermatitis. Clin Pediatr. 2019;58(4):406-412. doi:10.1177/ 0009922818825154

17. Gaddey HL, Holder K. Unintentional weight loss in older adults. $A m$ Fam Physician. 2014;89(9):718-722.

18. Melian EB, Spencer CM, Jarvis B. Clobetasol propionate foam, 0.05\%. Am J Clin Dermatol. 2001;2(2):89-92;discussion 93. doi:10.2165/00128071-200102020-00005

19. Broersen LH, Pereira AM, Jørgensen JO, Dekkers OM. Adrenal insufficiency in corticosteroids use: systematic review and meta-analysis. J Clin Endocrinol Metab. 2015;100(6):2171-2180. doi:10.1210/jc.2015-1218

20. Kerner M, Ishay A, Ziv M, Rozenman D, Luboshitzky R. Evaluation of the pituitary-adrenal axis function in patients on topical steroid therapy. J Am Acad Dermatol. 2011;65(1):215-216. doi:10.1016/j. jaad.2010.12.033

21. Malzfeldt E, Lehmann P, Goerz G, Lippold BC. Influence of drug solubility in the vehicle on clinical efficacy of ointments. Arch Dermatol Res. 1989;281(3):193-197. doi:10.1007/bf00456392

22. Manosroi W, Phimphilai M, Khorana J, Atthakomol P. Diagnostic performance of basal cortisol level at $0900-1300 \mathrm{~h}$ in adrenal insufficiency. PLoS One. 2019;14(11):e0225255. doi:10.1371/journal.pone. 0225255

23. Vogeser M, Kratzsch J, Ju Bae Y, et al. Multicenter performance evaluation of a second generation cortisol assay. Clin Chem Lab Med. 2017;55(6):826-835. doi:10.1515/cclm-2016-0400
International Journal of General Medicine

\section{Publish your work in this journal}

The International Journal of General Medicine is an international, peer-reviewed open-access journal that focuses on general and internal medicine, pathogenesis, epidemiology, diagnosis, monitoring and treatment protocols. The journal is characterized by the rapid reporting of reviews, original research and clinical studies across all disease areas. The manuscript management system is completely online and includes a very quick and fair peer-review system, which is all easy to use. Visit http://www.dovepress.com/ testimonials.php to read real quotes from published authors. 